\title{
Comets and Circumstellar Dust
}

\author{
C. Waelkens \\ Instituut voor Sterrenkunde K.U.Leuven, Celestijnenlaan 200B, 3001 \\ Leuven, Belgium \\ L.B.F.M. Waters \\ Sterrenkundig Instituut Anton Pannekoek, Kruislaan 403, $1098 \mathrm{SJ}$ \\ Amsterdam, The Netherlands
}

\begin{abstract}
Spectra obtained with the Infrared Space Observatory ISO of the dusty disks surrounding young stars display several characteristics which suggest the presence of large amounts of cometary material during these early stages. Of special interest are crystalline silicates and hydrated silicates, which surprisingly appear to be present already in cool material surrounding young objects. Another surprise is the occurrence of cometary features in the disks surrounding some binary post-AGB stars.
\end{abstract}

\section{Introduction}

The Kuiper-Edgeworth Belt and the Oort Cloud are considered to contain SolarSystem material which has been much less processed than in the inner Solar System, hence their interest as laboratories for the study of the early Solar System. The early evolution of planetary systems can also be studied from observations of the circumstellar disks of stars that were recently born.

Infrared spectroscopy of comets have revealed that silicates in comets are partially crystalline (e.g., Campins \& Ryan 1989, Hanner et al. 1990, Lynch et al. 1992), thus contrasting with the amorphous nature of silicates typically encountered in circumstellar and interstellar media. Among the rare objects in which crystalline silicate features also occur, are some young stars with dust debris disks, such as $\beta$ Pictoris (Knacke et al. 1993) and some Herbig Ae/Be stars (Hanner, Brooke \& Tokunaga 1998). Infrared spectroscopy then appears as a powerful tool for probing the role comets play in the early evolution of planetary systems.

In this context, ESA's Infrared Space Observatory ISO (Kessler et al. 1996) has considerably increased our observational potential, because it has opened a broad spectral window for spectroscopic observations, enabling us to probe the mineralogy of the circumstellar dust which is thought to become processed to comets, planetesimals, and ultimately planets. The sensitivity of ISO was too low to discover detailed spectral features in the debris disks of main-sequence stars such as Vega, but the spectrometers on board ISO have disclosed an unexpected, rich mineralogic variety in the more luminous disks of truly young stars, and especially so for Herbig Ae/Be stars, which are 2- to 8-solar-mass stars still evolving to or having recently reached the main sequence. In this contribution, 
we highlight the ISO results which appear to be most directly linked to Solar System studies.

\section{Crystalline silicates in circumstellar disks}

Before ISO, it was well known that interstellar and circumstellar absorption and emission spectra are often characterized by prominent silicate features around 10 and $18 \mu \mathrm{m}$. The typical broadness and smoothness of these bands show that 'astronomical silicates' are essentially amorphous. Substructure in the $10 \mu \mathrm{m}$ band, pointing to the presence of crystalline silicates as well, was documented then in a handful of objects, all of which were young stars with circumstellar disks, the most famous of which is $\beta$ Pictoris. Crystalline silicate features have been frequently observed, however, in the infrared spectra of Solar-System comets. Since in addition the frequent infall of comets onto the star is thought to be the explanation for the intermittent optical and ultraviolet absorption features observed for $\beta$ Pictoris and other objects, the study of crystalline features then becomes a promising tool for probing the evolutionary stages in circumstellar disks during which the Kuiper-Edgeworth Belt and the Oort Cloud are formed.

ISO disclosed that especially the spectral window between 28 and $70 \mu \mathrm{m}$, in which a true forest of crystalline silicate features was discovered, is suitable for such studies. A most fortunate circumstance was that the ISO era coincided with the appearance in the sky of Comet Hale-Bopp, the brightest and most dusty comet observed since decades (Crovisier et al. 1997). By chance, again, the spectrum of the Herbig Ae/Be star which is brightest in the infrared, i.e. HD100546 (Malfait et al. 1998, see Fig. 1), presents a remarkable similarity in its silicate features when compared to that of the comet. It should be noted, however, that this star has already spent some 5 million years on the main sequence, and that the silicate emission for the younger Herbig $\mathrm{Ae} / \mathrm{Be}$ stars tends to be more dominated by amorphous grains (e.g. van den Ancker et al. 2000).

The prominence of strong crystalline silicate emission in the disks of young circumstellar disks suggests that the partial crystallinity of the silicates in comets originates from the early epochs of their formation, and has not been acquired during recent passages close to the Sun. It is not known, however, what is the cause which triggers the crystallization process. The most obvious explanation, which is thermal annealing (Hallenbeck \& Nuth 1998), does not appear to be consistent with the low temperatures at which the crystalline silicates are observed to occur. Interestingly, crystalline silicates are also prominent in the circumstellar environments of several very evolved objects (Molster et al. 1999), most often in an evolutionary stage after the asymptotic giant branch (AGB). It appears that the objects with the strongest crystalline features are components of binaries, the circumstellar dust of which resides in a long-lived circum-binary disk. This observation may hint to the fact that time is an essential parameter in the process, but may also underscore that comet or planetesimal formation is a rather natural phenomenon in long-lived disks of sufficient density. By comparing the strengths of the crystalline features with the relative emission of disks at $\mathrm{mm}$ wavelengths, Molster et al. (1999) showed that crystallization is highest in objects for which the energy distribution points to the presence of large grains, hence confirming that the occurrence of crystalline silicates is a reliable indicator of the processing of circumstellar disks. 


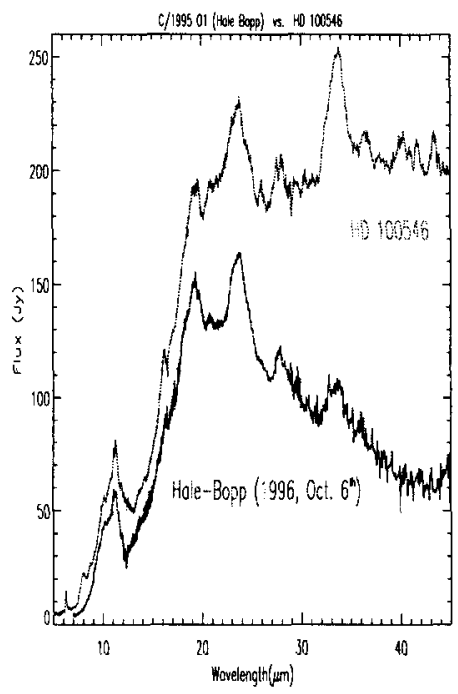

Figure 1. Comparison of the ISO-SWS spectra of the Herbig Ae/Be star HD100546 and of Comet Hale-Bopp. The similarity of the crystalline forsterite features in both objects is striking. (Figure from Malfait et al. 1998)

\section{Water ice and hydrous silicates in the disks of young stars}

The presence of water ice has been advocated as an explanation of the high reflectance of the $\beta$ Pictoris disk (Pantin, Lagage \& Artymovicz 1997), but ISO observations of this object were not able to confirm this prediction. On the other hand, spectroscopic evidence has been found for important amounts of water ice in the disks of several Herbig Ae/Be stars, such as HD100546 and HD142527 (Malfait et al. 1999). A remarkable result is that most of this water ice is crystalline, apparently conflicting with the low temperatures of the disks. The case of HD142527 is extreme: for this object, nearly $60 \%$ of the infrared emission is due to water ice, of which $90 \%$ is crystalline; moreover, the temperature of the disk is only between 30 and $60 \mathrm{~K}$, and the very young age of the object does not appear to allow for significant outwards migration of warmer ices.

In this same object HD142527, as well as in several others, among which HD100546, a broad shoulder between 90 and $110 \mu \mathrm{m}$ is observed, which can convincingly be modeled with the available optical constants of hydrous silicates. The occurrence in meteorites of such silicates with water-ice inclusions is well known (Wilkening 1978). It is now widely accepted that hydrous inclusions are extraterrestrial in origin, and not the result of terrestrial contamination (Zolensky \& McSween 1988). The detection of hydrous silicates in young circumstellar disks confirms this hypothesis, and forms a nice confirmation of the stimulating connections which exist between Solar-System science and stellar astronomy. 


\section{Concluding remarks}

Infrared spectroscopy from space has opened the field of circumstellar mineralogy, for the benefit of both the Solar-System and the stellar communities. It also has had a stimulating effect on new developments in laboratory astrophysics, to synthesize laboratory analogs for cosmic dust and to provide infrared spectroscopists with realistic optical constants with which to model circumstellar spectra.

Unfortunately, the sensitivity of the ISO instruments was not sufficient to observe a sufficient amount of objects in order to cover accurately the whole apparent diversity of the faint disks surrounding young stars. The NASA SIRTF mission will provide us with a significant increase of the sample, albeit not with as large a wavelength coverage as provided by ISO.

\section{References}

Campins, H., Ryan, E.V. 1989, ApJ, 341, 1059

Crovisier, J., Leech, K., Bockelée-Morvan, D., Brooke, T.Y., Hanner, M.S., Altieri, B., Keller, H.U., Lellouch, E. 1997, Science, 275, 1904

Hallenbeck, S., Nuth, J. 1998, Ap\&SS, 255, 427

Hanner, M.S., Brooke, T.Y., Tokunaga, A.T. 1998, ApJ, 502, 871

Hanner, M.S., Newburn, R. L., Gehrz, R. D., Harrison, T., Ney, E. P., Hayward, T. L. 1990, ApJ, 348, 312

Kessler, M.F., Steinz, J. A., Anderegg, M. E., Clavel, J., Drechsel, G., Estaria, P., Faelker, J., Riedinger, J. R., Robson, A., Taylor, B. G., Ximenez de Ferran, S. 1996, A\&A, 315, L27

Knacke, R.F., Fajardo-Acosta, S. B., Telesco, C. M., Hackwell, J. A., Lynch, D. K., Russell, R. W. 1993, ApJ, 418, 440

Lynch, D.K., Russell, R. W., Hackwell, J. A., Hanner, M. S., Hammel, H. B. 1992, Icarus, 100, 197

Malfait, K., Waelkens, C., Waters, L. B. F. M., Vandenbussche, B., Huygen, E., de Graauw, M. S. 1998, A\&A, 332, L25

Malfait, K., Waelkens, C., Bouwman, J., de Koter, A., Waters, L. B. F. M., 1999, A\&A, 345, 181

Molster, F.J., Yamamura, I., Waters, L. B. F. M., Tielens, A. G. G. M., de Graauw, Th., de Jong, T., de Koter, A., Malfait, K., van den Ancker, M. E., Van Winckel, H., Voors, R. H. M., Waelkens, C. 1999, Nature, 401, 563

Pantin, E., Lagage, P.O., Artymovicz, P. 1997, A\&A, 327, 1123

van den Ancker, M.E., Bouwman, J., Wesselius, P. R., Waters, L. B. F. M., Dougherty, S. M., van Dishoeck, E. F. 2000, A\&A, 357, 325

Wilkening, L.L. 1978, Naturwissenschaften, 65, 73

Zolensky, M., McSween, H.Y. 1988, in Meteorites and the Early Solar System, J.F. Kerridge \& M.S. Matthews, eds. (Tucson: U. Arizona Press), 114 